QUARTERLY OF APPLIED MATHEMATICS

VOLUME LXV, NUMBER 2

JUNE 2007, PAGES 259-279

S $0033-569 X(07) 01051-2$

Article electronically published on April 19, 2007

\title{
CONSERVATIVE HYPERBOLIC FORMULATION FOR COMPRESSIBLE TWO-PHASE FLOW WITH DIFFERENT PHASE PRESSURES AND TEMPERATURES
}

\author{
BY \\ E. ROMENSKI (Sobolev Institute of Mathematics, Novosibirsk 630090, Russia), \\ A. D. RESNYANSKY (DSTO, P.O. Box 1500, Edinburgh SA 5111, Australia), \\ AND \\ E. F. TORO (University of Trento, Trento, 38050, Italy)
}

\begin{abstract}
Governing equations for two-phase compressible flow with different phase pressures and temperatures are presented, the derivation of which is based on the formalism of thermodynamically compatible hyperbolic systems and extended irreversible thermodynamics principles. These equations form a hyperbolic system in conservationlaw form. A two-phase isentropic flow model proposed earlier and the hyperbolic model for heat transfer underlie the developed theory of this paper. A set of interfacial exchange processes such as pressure relaxation, interfacial friction, temperature relaxation and phase transition is taken into account by source terms in the balance equations. It is shown that the heat flux relaxation limit of the governing equations can be written in the Baer-Nunziato form, in which the Fourier thermal conductivity diffusion terms for each phase are included.
\end{abstract}

1. Introduction. Multiphase flow modelling has undergone intensive developments in recent years because of its great importance for practical applications. In recent decades several theories of two-phase flow have been developed and exploited successfully for the needs of industry and environmental sciences.

In this paper we study a model of two-phase two-fluid flow in which the constituents of the mixture have different velocities, pressures and temperatures. Mathematical models for such a flow are still far from a final satisfactory state. One of the most popular models

Received August 29, 2005.

2000 Mathematics Subject Classification. Primary 35L65, 76T99.

Current address: Cranfield University, Cranfield MK43 0AL, UK.

E-mail address: evrom@math.nsc.ru

E-mail address: Anatoly.Resnyansky@dsto.defence.gov.au

E-mail address: toro@ing.unitn.it 
in this field has been proposed by Baer and Nunziato 2. The governing equations of the model are taken as a set of two separate systems of conservation laws for each phase coupled by interface exchange terms. This model has been applied to the study of many concrete problems, and some modifications and generalizations of this model have been made in, for example, [1, 3, 8, 16, 24] and the references therein. Note that the governing equations of the Baer-Nunziato model are hyperbolic. This is a very important attribute of the model, in that it gives a theoretical basis for its mathematical study and for confidence in numerical solutions of the equations. But not all equations of the BaerNunziato type model are in conservative form, and this results in difficulties in defining and studying discontinuous solutions [13, 25].

We propose a different approach to model two-phase flows, based on the formalism of thermodynamically compatible systems. This theory allows us to formulate classes of hyperbolic conservation equations using generating thermodynamic potentials and variables. Its core is a phenomenological approach in modelling of continuous media and using thermodynamic laws, which determine the structure of the governing equations. The theory of thermodynamically compatible systems has been developed in the last few decades and has been successfully applied in different areas of continuum mechanics and mathematical physics 7, 9, 12, 18. With respect to multiphase compressible flow a general principle has been constructed [21, 22, and the model for two-phase flows in which thermal nonequilibrium between the phases is negligible has been developed and applied to the study of some specific problems [19, 20, 23. In this approach the mixture is supposed to be a continuum in which the two-phase character of the flow is taken into account. The complete set of governing equations is a hyperbolic system and consists of the mass, momentum and energy conservation laws for the mixture, which are completed by balance laws for additional mixture parameters, namely, for the volume fraction and relative velocity. Moreover, all equations of the system have a conservative form. This fact is very attractive from mathematical and numerical viewpoints, because it gives a straightforward way to develop the theory of discontinuous solutions and to apply modern numerical methods. Note that the equations of the conservative model can be transformed to a system that is similar to the Baer-Nunziato form. The difference consists of the distinct definition of interfacial pressure and lift forces that appear in the phase momentum equations. These forces are not included into the traditional BaerNunziato model.

In this paper we present a generalization of the model 23 for the case of flow with two different phase temperatures. The design methodology of the model is based on the synthesis of the conservative equations from [23] and hyperbolic heat transfer equations formulated in [17. The hyperbolic equations for heat conductivity are well-known and widely discussed in the literature; see for example [18. This model is based on the Cattaneo equation for heat transfer, and the temperature and heat flux are used as variables. In the present paper we use phase entropies and entropy fluxes as physical variables which seems to be more convenient for our study and are typical variables in irreversible thermodynamics [5]. The resulting system of governing equations is also hyperbolic and all equations are written in conservative form. 
The model presented in the paper is designed for two-phase flows in which both phases are compressible and the relative motion is of a considerable magnitude. We note that the model is supposed to be a basic model and for each concrete flow the closure constitutive relations (equation of state, source terms) should be specified by taking into account features of constituents and their interaction. The model's possible areas of application include gas/liquid flows (liquid droplets in gas or gas bubbles in liquid) and solid particles/gas flows at high pressure. The temperature and heat transfer rate in these types of flow can be pretty large. It is necessary to note that the heat transfer characteristic relaxation time in pure liquid or gas is extremely short and the applicability of a hyperbolic law for heat transfer instead of the parabolic Fourier law could be reasonable for modelling processes with a comparable characteristic time. In our case of two-phase flow the mechanism of heat transfer is determined mainly by the relative motion of phases and whether the characteristic time of such a mechanism of heat transfer is large. In Sect. 4we will see that the proposed hyperbolic model takes into account this feature of the flow.

The paper is organized as follows. In Sect. 2 we give a brief formulation of the conservative model for compressible two-phase flow, in which thermal effects are ignored. Sect. 3 describes the hyperbolic heat transfer equations, on the basis of which the sophisticated conservative model for two-phase flow with two temperatures is presented in Sect. 4. Also in Sect. 4 the diffusive limit of the hyperbolic heat flux relaxation is discussed. It is shown that such an approximation leads to equations similar to the Baer-Nunziato equations with parabolic phase thermal conductivity terms. Conclusions are drawn in Sect. 5. Finally, in the Appendix, a description of the generating system is given, which is formulated by the thermodynamically compatible systems theory and generates the proposed two-phase model.

2. Two-phase flow equations in the case of negligible temperature variations. In this section we briefly review the model for two-phase flow proposed in [23]. Its derivation is based on the principles of thermodynamically compatible systems [12, and the governing equations form a hyperbolic system of differential equations in conservative form. We ignore here many possible dissipative processes and take into account only interfacial friction and relaxation of phase pressures to the common pressure value. Variations of phase temperatures are assumed to be negligible, and in this section we ignore thermal processes.

We consider processes in the Cartesian coordinate system, the tensor notation such as upper and lower indexes, and summation with respect to common index are used. Note that sometimes we identify quantities with lower and upper indexes in order to avoid multiple indexation. Assume that the mixture is a continuum in which the two-phase character of the flow is taken into account. Suppose that the state of each phase with a number $i=1,2$ is characterized by its volume fraction $\alpha_{i}$, mass density $\rho_{i}$ and velocity vector $u_{i}^{k}, k=1,2,3$. For the volume fractions the saturation constraint $\alpha_{1}+\alpha_{2}=1$ is assumed.

The set of convenient physical parameters of state for the derivation of the governing equations is $\alpha, \rho, c, u^{k}, w^{k}$, where $\alpha=\alpha_{1}$ is the volume fraction of the first phase, $\rho$ is 
the mixture mass density, $c=c_{1}$ is the mass fraction of the first phase, $u^{k}$ is the average velocity of the mixture, and $w^{k}$ is the relative velocity of phases.

These parameters of state for the mixture are connected with the parameters of state for each phase by the relations

$$
\alpha=\alpha_{1}, \rho=\alpha_{1} \rho_{1}+\alpha_{2} \rho_{2}, c=\frac{\alpha_{1} \rho_{1}}{\rho}, u^{k}=c_{1} u_{1}^{k}+c_{2} u_{2}^{k}, w^{k}=u_{1}^{k}-u_{2}^{k}
$$

Using (2.1) we can derive the relations expressing the individual parameters, such as mass densities and velocities, by the mixture parameters:

$$
\begin{aligned}
& \rho_{1}=\frac{c_{1} \rho}{\alpha_{1}}=\frac{c \rho}{\alpha}, \quad \rho_{2}=\frac{c_{2} \rho}{\alpha_{2}}=\frac{(1-c) \rho}{(1-\alpha)} \\
& u_{1}^{k}=u^{k}+c_{2} w^{k}=u^{k}+(1-c) w^{k}, \quad u_{2}^{k}=u^{k}-c_{1} w^{k}=u^{k}-c w^{k} .
\end{aligned}
$$

The system of governing equations for two-phase flow written in terms of the mixture parameters (2.1) can be derived with the formalism of thermodynamically compatible systems (see Appendix) and reads as follows:

$$
\begin{aligned}
& \frac{\partial \rho \alpha}{\partial t}+\frac{\partial \rho u^{k} \alpha}{\partial x^{k}}=-\phi \\
& \frac{\partial \rho}{\partial t}+\frac{\partial \rho u^{k}}{\partial x^{k}}=0 \\
& \frac{\partial \rho u^{l}}{\partial t}+\frac{\partial\left(\rho u^{l} u^{k}+p \delta^{k l}+\rho w^{l} E_{w_{k}}\right)}{\partial x^{k}}=0, \\
& \frac{\partial \rho c}{\partial t}+\frac{\partial\left(\rho u^{k} c+\rho E_{w_{k}}\right)}{\partial x^{k}}=0, \\
& \frac{\partial w_{k}}{\partial t}+\frac{\partial\left(u^{l} w_{l}+E_{c}\right)}{\partial x^{k}}=-\left(e_{k l j} u^{l} \omega^{j}+\lambda_{k}\right) .
\end{aligned}
$$

The equations of the above system are the balance laws for volume fraction, total mass, total momentum, mass fraction and relative velocity, respectively. Here $e_{k l j}$ is the unit pseudoscalar. Fluxes in the last three equations contain the derivatives of the equation of state (specific internal energy) $E$, which is supposed to be a known function of the parameters of state. The momentum flux contains the mixture pressure $p$, which is also defined by the derivative of the equation of state with respect to the mixture density:

$$
p=\rho^{2} E_{\rho}=\rho^{2} \frac{\partial E}{\partial \rho} .
$$

The two source terms in the first and in the last equations of system (2.4) are the pressure relaxation term and interfacial friction term, respectively. The variable $\omega_{j}$ is an 
auxiliary variable and its introduction is necessary to write the equation for the relative velocity in conservative form. Therefore, two additional equations should be added to the system (2.4) in order to provide its compatibility [22]. The first one is a steady conservative equation

$$
e^{j k l} \frac{\partial w_{k}}{\partial x^{l}}=\omega^{j},
$$

which defines $\omega_{i}$ as the vorticity of the relative velocity vector, and the second one is as follows:

$$
\frac{\partial \omega^{k}}{\partial t}+\frac{\partial\left(u^{l} \omega_{k}-u^{k} \omega^{l}+e^{k l j} \lambda_{j}\right)}{\partial x^{l}}=0
$$

We emphasize again that the vector variable $\omega^{i}$ is introduced only to write the equation for the relative velocity in conservative form. Actually one can use in system (2.4) the equivalent nonconservative equation for the relative velocity

$$
\frac{\partial w_{k}}{\partial t}+u^{l} \frac{\partial w_{k}}{\partial x^{l}}+\frac{\partial E_{c}}{\partial x^{k}}+w_{l} \frac{\partial u^{l}}{\partial x^{k}}=-\lambda_{k}
$$

instead of the conservative one. Nevertheless, the additional Eqs. (2.6) and (2.7) can be useful in studying discontinuous solutions.

It is reasonable to define the source terms in the following way:

$$
\phi=\frac{\rho}{\tau^{(p)}} E_{\alpha}=\frac{\rho}{\tau^{(p)}} \frac{\partial E}{\partial \alpha}, \quad \lambda_{k}=\zeta E_{w^{k}}=\zeta \frac{\partial E}{\partial w^{k}},
$$

where $\tau^{(p)}$ is the pressure relaxation time which is assumed to be a function of parameters of state and $\zeta$ is the interfacial friction coefficient, which also can be a function of parameters of state. Further we will see that such a definition will provide the positiveness of entropy production in more sophisticated models of two-phase flow.

Thus the closing relations for the system (2.4) are the source terms $\phi, \lambda_{k}$ and the equation of state $E$. The source terms are defined by formulae (2.9) in which the derivatives of the equation of state $E_{\alpha}, E_{w^{k}}$ are included. Only the pressure relaxation time $\tau^{(p)}$ and the interfacial friction coefficient $\zeta$ should be defined empirically using physical assumptions and experimental data.

Now we describe how to define the equation of state for the mixture if the equations of state for each phase are known. First of all, we assume that the equation of state for the mixture has a well-known form [26], and is represented as the sum of the specific internal energy of the mixture $e$ and the kinematic energy of the relative motion:

$$
E\left(\alpha, \rho, c, w_{1}, w_{2}, w_{3}\right)=e(\alpha, \rho, c)+c(1-c) \frac{w_{i} w^{i}}{2} .
$$


Note that the introduction of the kinematic energy of the relative motion into the equation of state is a consequence of the requirement that the total kinetic energy of the mixture is the sum of phase partial kinetic energies:

$$
\rho \frac{u_{i} u^{i}}{2}+\rho c(1-c) \frac{w_{i} w^{i}}{2}=\alpha_{1} \rho_{1} \frac{u_{1}^{i} u_{1}^{i}}{2}+\alpha_{2} \rho_{2} \frac{u_{2}^{i} u_{2}^{i}}{2} .
$$

Let us suppose now that the equation of state (specific internal energy) for each phase is a known function of its mass density $e^{i}=e^{i}\left(\rho_{i}\right),(i=1,2)$. Let us assume in addition that

$$
e(\alpha, \rho, c)=c_{1} e^{1}\left(\rho_{1}\right)+c_{2} e^{2}\left(\rho_{2}\right) .
$$

Using the relations (2.2) connecting phase mass densities and parameters of the mixture, we obtain

$$
e(\alpha, \rho, c)=c e^{1}\left(\frac{c \rho}{\alpha}\right)+(1-c) e^{2}\left(\frac{(1-c) \rho}{(1-\alpha)}\right),
$$

which gives us the internal energy for the mixture.

The definition of the equation of state for the mixture (2.10), (2.12) via equations of state for each phase gives us the possibility of rewriting the system (2.4) in terms of individual parameters of each phase [23. We do not reproduce the conservative system in terms of phase individual parameters here because such a derivation will be done later for a more sophisticated model with two temperatures.

The system (2.4) can be rewritten in terms of phase individual parameters in the Baer-Nunziato type form as it is, for example, in [24, in which two sets of balance equations for each phase are coupled by the interfacial momentum exchange terms. The transformation of the equations is not difficult, but it is a cumbersome procedure. We provide the final system only, which is as follows:

$$
\begin{aligned}
& \frac{\partial \rho \alpha_{1}}{\partial t}+\frac{\partial \rho \alpha_{1} u^{k}}{\partial x^{k}}=\frac{p^{1}-p^{2}}{\tau}, \\
& \frac{\partial \alpha_{1} \rho_{1}}{\partial t}+\frac{\partial \alpha_{1} \rho_{1} u_{1}^{k}}{\partial x^{k}}=0, \\
& \frac{\partial \alpha_{2} \rho_{2}}{\partial t}+\frac{\partial \alpha_{2} \rho_{2} u_{2}^{k}}{\partial x^{k}}=0, \\
& \frac{\partial \alpha_{1} \rho_{1} u_{1}^{i}}{\partial t}+\frac{\partial \alpha_{1} \rho_{1} u_{1}^{i} u_{1}^{k}}{\partial x^{k}}+\frac{\partial \alpha_{1} p^{1}}{\partial x^{i}}=\hat{p}_{I} \frac{\partial \alpha_{1}}{\partial x^{i}}+f_{i}+\hat{\zeta}\left(u_{2}^{i}-u_{1}^{i}\right), \\
& \frac{\partial \alpha_{2} \rho_{2} u_{2}^{i}}{\partial t}+\frac{\partial \alpha_{2} \rho_{2} u_{2}^{i} u_{2}^{k}}{\partial x^{k}}+\frac{\partial \alpha_{2} p^{2}}{\partial x^{i}}=\hat{p}_{I} \frac{\partial \alpha_{2}}{\partial x^{i}}-f_{i}-\hat{\zeta}\left(u_{2}^{i}-u_{1}^{i}\right) .
\end{aligned}
$$

Here, as before, $\rho=\alpha_{1} \rho_{1}+\alpha_{2} \rho_{2}, u^{k}=\frac{\alpha_{1} \rho_{1}}{\rho} u_{1}^{k}+\frac{\alpha_{2} \rho_{2}}{\rho} u_{2}^{k}, \hat{\zeta}$ is the modified interfacial friction coefficient

$$
\hat{\zeta}=\zeta \frac{\left(\alpha_{1} \alpha_{2} \rho_{1} \rho_{2}\right)^{2}}{\rho^{3}}
$$


and $\hat{p}_{I}$ is an interfacial pressure:

$$
\hat{p}_{I}=\frac{\alpha_{2} \rho_{2} p^{1}+\alpha_{1} \rho_{1} p^{2}}{\alpha_{1} \rho_{1}+\alpha_{2} \rho_{2}} .
$$

The definition of interfacial pressure in our model differs from the definition in the conventional Baer-Nunziato type models; for example, $p_{I}=\alpha_{1} p_{1}+\alpha_{2} p_{2}$ in [24]. The more significant difference are the terms $f_{i}$ in the momentum equations, which do not appear in the Baer-Nunziato model:

$$
f_{i}=\rho c_{1} c_{2}\left(u_{1}^{k}-u_{2}^{k}\right)\left(c_{1}\left(\frac{\partial u_{2}^{i}}{\partial x^{k}}-\frac{\partial u_{2}^{k}}{\partial x^{i}}\right)+c_{2}\left(\frac{\partial u_{1}^{i}}{\partial x^{k}}-\frac{\partial u_{1}^{k}}{\partial x^{i}}\right)\right) .
$$

These forces arise for the flow with nonzero relative velocity and are caused by the phase vorticities. Such type of force is called lift force; see, for example, [6].

3. Hyperbolic heat transfer equations. The model for two-phase compressible flow with two temperatures proposed in this paper uses a hyperbolic heat transfer approach, which gives a finite speed of propagation of thermal perturbations. Such an approach in heat transfer modelling was discovered by Cattaneo and has been studied by many researchers; see, for example, [18. The Cattaneo theory is usually formulated in terms of temperature $T$ and heat flux $J_{k}$, and the governing equation for $J_{k}$ is as follows:

$$
\tau \frac{\partial J_{k}}{\partial t}+\kappa \frac{\partial T}{\partial x^{k}}=-J_{k}
$$

Here $\kappa$ is the thermal conductivity coefficient, and $\tau$ is the heat flux relaxation time.

For our purpose another set of variables is preferable, namely, entropy and entropy flux. These variables, accepted in irreversible thermodynamics [5, have been used in [17. We also presuppose the existence of a thermodynamic potential-specific internal energy $E$. Moreover, we assume that the entropy flux is a derivative of the equation of state (specific internal energy) with respect to the conjugate variable which we call the thermal impulse. Such a set of variables including the entropy and thermal impulse seem to be more convenient for models of complicated media. Finally, we assume that heat transfer processes are governed by the following system:

$$
\begin{aligned}
& \rho \frac{\partial S}{\partial t}+\frac{\partial E_{j^{k}}}{\partial x^{k}}=\sigma, \\
& \rho \frac{\partial j_{k}}{\partial t}+\frac{\partial T}{\partial x^{k}}=-\frac{E_{j^{k}}}{\kappa} .
\end{aligned}
$$

Here $\rho$ is the mass density of the medium, which is assumed to be incompressible, $S$ is the specific entropy, $j_{k}$ is the thermal impulse, $E\left(S, j_{k}\right)$ is the specific internal energy, $T=E_{S}$ is the temperature, and $\sigma$ is the entropy production. To close the system (3.2) it is necessary to define the dependence of internal energy on $S$ and $j^{k}$. The simplest one we can choose is

$$
E=e(S)+\frac{A}{2} j_{k} j^{k}, \quad A=\frac{\rho \kappa}{\tau} .
$$

The term $e(S)$ can be derived supposing that the medium is in equilibrium state $\left(j^{k}=0\right)$ and by integrating the equation $d e=C(T) d T=T d S$, where $C(T)$ is the specific heat 
capacity. Note that if we denote $j^{k}=\frac{\tau}{\rho \kappa} J^{k}$ and take $A=\frac{\rho \kappa}{\tau}$ we obtain for $J^{k}$ Eq. (3.1) directly.

Multiplying the equations of the system (3.2) by $E_{S}$ and $E_{j_{k}}$ respectively and summing the results, we obtain the energy balance law in the form

$$
\rho \frac{\partial E}{\partial t}+\frac{\partial E_{S} E_{j_{k}}}{\partial x^{k}}=\sigma E_{S}-\frac{E_{j_{k}} E_{j^{k}}}{\kappa} .
$$

From the first law of thermodynamics, namely, the conservation of energy, we conclude that the right-hand side in the latter equation must be zero and hence for the entropy production we have

$$
\sigma=\frac{1}{T} \frac{E_{j_{k}} E_{j^{k}}}{\kappa} \geq 0 .
$$

It is important to note that the classical Fourier heat transfer law can be obtained assuming that the time scale of the processes under consideration is much bigger than $\tau$. Appealing to physical intuition we do not provide a proof of this statement. Some mathematical results concerning the relaxation limit of hyperbolic equations can be found in [4, 15. So the relaxation limit can be obtained by neglecting the term $\partial j_{k} / \partial t$ in the second equation of the system (3.2), and the entropy flux becomes

$$
E_{j^{k}}=-\kappa \frac{\partial T}{\partial x^{k}} .
$$

Hence the entropy and energy equations take the form

$$
\begin{gathered}
\rho \frac{\partial S}{\partial t}-\frac{\partial}{\partial x^{k}}\left(\kappa \frac{\partial T}{\partial x^{k}}\right)=\kappa \frac{1}{T} \sum_{k=1}^{3}\left(\frac{\partial T}{\partial x^{k}}\right)^{2}, \\
\rho \frac{\partial E}{\partial t}-\frac{\partial}{\partial x^{k}}\left(\kappa T \frac{\partial T}{\partial x^{k}}\right)=0 .
\end{gathered}
$$

These equations are well-known in heat transfer Fourier theory.

4. Conservative model for two-phase flow with two temperatures. Now we can construct a more sophisticated model for the flow of the mixture of two compressible phases with different velocities, pressures and temperatures. The model is based on the synthesis of two models presented in previous sections - the isentropic model for two-phase flow and the hyperbolic equations for heat transfer. We shall consider mechanisms such as interfacial exchange, pressure relaxation, interfacial friction, interfacial temperature exchange and phase transition.

4.1. Generating system of conservative equations. First of all we define the set of parameters of state for each phase

$$
\alpha_{i}, \quad \rho_{i}, \quad s_{i}, \quad u_{i}^{k}, \quad j_{i}^{k},
$$

where $i$ is the phase number $(i=1,2), \alpha_{i}$ is the volume concentration of the $i$-th phase, $\rho_{i}$ is the mass density of the $i$-th phase, $s_{i}$ is the specific entropy of the $i$-th phase, $u_{i}^{k}$ is the velocity vector of the $i$-th phase, and $j_{i}^{k}$ is the specific thermal impulse of the $i$-th phase. It is supposed that the saturation constraint for volume fractions $\alpha_{1}+\alpha_{2}=1$ holds. 
The theory of thermodynamically compatible system allows us to formulate the system of governing equations in terms of parameters of state for the mixture:

$$
\begin{gathered}
\alpha_{1}, \quad \rho=\alpha_{1} \rho_{1}+\alpha_{2} \rho_{2}, \quad c_{1}=\frac{\alpha_{1} \rho_{1}}{\rho}, \\
u^{k}=c_{1} u_{1}^{k}+c_{2} u_{2}^{k}, \quad w^{k}=u_{1}^{k}-u_{2}^{k}, \quad S_{i}=c_{i} s_{i}, \quad j_{i}^{k} .
\end{gathered}
$$

Here $\rho$ is the total mass density of the mixture, $c_{i}$ is the mass concentration of the $i$-th phase, $u^{k}$ is the mixture velocity vector, $w^{k}$ is the relative velocity vector, $S_{i}$ is the partial entropy of the $i$-th phase, and $j_{i}^{k}$ is the thermal impulse vector of the $i$-th phase. It is obvious that the saturation constraint $c_{1}+c_{2}=1$ is also valid for phase mass fractions.

As in Sect. 2 let us denote $\alpha=\alpha_{1}, c=c_{1}$. The basic system of governing equations can be derived from the generating system of conservation laws (see Appendix) and reads as follows:

$$
\begin{aligned}
& \frac{\partial \rho}{\partial t}+\frac{\partial \rho u^{k}}{\partial x^{k}}=0 \\
& \frac{\partial \rho \alpha}{\partial t}+\frac{\partial \rho \alpha u^{k}}{\partial x^{k}}=-\phi, \\
& \frac{\partial \rho c}{\partial t}+\frac{\partial\left(\rho c u^{k}+E_{w_{k}}\right)}{\partial x^{k}}=-\psi, \\
& \frac{\partial \rho u^{i}}{\partial t}+\frac{\partial\left(\rho u^{i} u^{k}+p \delta^{i k}+\rho w^{i} E_{w_{k}}\right)}{\partial x^{k}}=0, \\
& \frac{\partial w_{k}}{\partial t}+\frac{\partial\left(u^{l} w_{l}+E_{c}\right)}{\partial x^{k}}=-e_{k l j} u^{l} \omega^{j}-\frac{1}{\rho} \lambda_{0}^{k}, \\
& \frac{\partial \rho j_{1}^{i}}{\partial t}+\frac{\partial\left(\rho j_{1}^{i} u^{k}+E_{S_{1}} \delta^{i k}\right)}{\partial x^{k}}=-\lambda_{1}^{i}, \\
& \frac{\partial \rho j_{2}^{i}}{\partial t}+\frac{\partial\left(\rho j_{2}^{i} u^{k}+E_{S_{2}} \delta^{i k}\right)}{\partial x^{k}}=-\lambda_{2}^{i}, \\
& \frac{\partial \rho S_{1}}{\partial t}+\frac{\partial\left(\rho S_{1} u^{k}+E_{j_{1}^{k}}\right)}{\partial x^{k}}=\Pi_{1}-\pi_{1}, \\
& \frac{\partial \rho S_{2}}{\partial t}+\frac{\partial\left(\rho S_{2} u^{k}+E_{j_{2}^{k}}\right)}{\partial x^{k}}=\Pi_{2}-\pi_{2} .
\end{aligned}
$$

The equations of system (4.2) are the total mass conservation law, the balance law for the volume fraction, the balance law for the mass fraction, the total momentum conservation law, the balance law for the relative velocity, two balance laws for phase thermal impulses, and two balance laws for the phase entropies, respectively. The pressure $p$ is defined in the same way as in Sect. 2. $p=\rho^{2} E_{\rho}$.

The constitutive relations which should be defined for the closure of system (4.2) are the specific internal energy $E$ and the source terms $\phi, \lambda_{0}^{k}, \lambda_{1}^{i}, \lambda_{2}^{i}, \lambda_{3}^{i}, \Pi_{1}, \Pi_{2}, \pi_{1}, \pi_{2}$. 
The procedure of derivation of $E$ by the known equations of state for each phase is similar to Sect. 2 and will be given below. The source terms are defined in the following way (note that our goal is not to describe a possible general structure of source terms, but to select only those that are essential for the modelling of physical processes in which we are interested):

$$
\begin{gathered}
\phi=\frac{\rho}{\tau^{(p)}} E_{\alpha}, \\
\psi=\frac{\rho}{\tau^{(c)}} E_{c}, \\
\lambda_{0}^{k}=\chi_{00} E_{w_{k}}+\chi_{01} E_{j_{1}^{k}}+\chi_{02} E_{j_{2}^{k}}, \quad \lambda_{1}^{k}=\chi_{01} E_{w_{k}}+\chi_{11} E_{j_{1}^{k}}, \quad \lambda_{2}^{k}=\chi_{02} E_{w_{k}}+\chi_{22} E_{j_{2}^{k}}, \\
\Pi_{1}=\frac{1}{E_{S_{1}}} \chi_{11} \sum_{k=1}^{3}\left(E_{j_{1}^{k}}+\frac{\chi_{01}}{\chi_{11}} E_{w^{k}}\right)^{2}+\frac{c_{1}}{E_{S_{1}}}\left(\chi_{00}-\frac{\chi_{01}^{2}}{\chi_{11}}-\frac{\chi_{02}^{2}}{\chi_{22}}\right) \sum_{k=1}^{3}\left(E_{w^{k}}\right)^{2} \\
+\frac{\rho c_{1}}{E_{S_{1}}} \frac{E_{\alpha}^{2}}{\tau^{(p)}}+\frac{\rho c_{1}}{E_{S_{1}}} \frac{E_{c}^{2}}{\tau^{(c)}}, \\
\Pi_{2}=\frac{1}{E_{S_{2}}} \chi_{22} \sum_{k=1}^{3}\left(E_{j_{2}^{k}}+\frac{\chi_{02}}{\chi_{22}} E_{w^{k}}\right)^{2}+\frac{c_{2}}{E_{S_{2}}}\left(\chi_{00}-\frac{\chi_{01}^{2}}{\chi_{11}}-\frac{\chi_{02}^{2}}{\chi_{22}}\right) \sum_{k=1}^{3}\left(E_{w^{k}}\right)^{2} \\
+\frac{\rho c_{2}}{E_{S_{2}}} \frac{E_{\alpha}^{2}}{\tau^{(p)}}+\frac{\rho c_{2}}{E_{S_{2}}} \frac{E_{c}^{2}}{\tau^{(c)}}, \\
\pi_{1}=\frac{1}{\tau^{(T)}} \frac{E_{S_{1}}-E_{S_{2}}}{E_{S_{1}}}, \\
\pi_{2}=\frac{1}{\tau^{(T)}} \frac{E_{S_{2}}-E_{S_{1}}}{E_{S_{2}}} .
\end{gathered}
$$

These source terms are responsible for the following physical processes: $\phi$ governs the relaxation of phase pressures to a common uniform state, $\psi$ governs the rate of phase transition, $\lambda_{0}^{k}$ simulate the interfacial friction force, and $\lambda_{1}^{k}$ and $\lambda_{2}^{k}$ simulate relaxation of the phase thermal impulses to the corresponding equilibrium states. Here a mutual dependence of thermal impulses and relative velocity is taken into account. We also emphasize that coefficients $\chi_{i j}$ in the formulae for $\lambda_{j}^{i}$ are taken as symmetric, which is a consequence of the Onsager principle [5]. $\Pi_{1}$ and $\Pi_{2}$ simulate the phase entropy production caused by all of the above-mentioned relaxation processes, and finally $\pi_{1}$ and $\pi_{2}$ govern the phase energy exchange leading to equalizing of the temperatures of the two phases.

We emphasize that the source terms $\Pi_{1}, \Pi_{2}$ in the equations for partial entropies must be nonnegative and hence the coefficients

$$
\left(\chi_{00}-\frac{\chi_{01}^{2}}{\chi_{11}}-\frac{\chi_{02}^{2}}{\chi_{22}}\right), \quad \chi_{11}, \quad \chi_{22}, \quad \tau^{(p)}, \quad \tau^{(c)}
$$

must be nonnegative. We also suppose that $\tau^{(T)}$ is nonnegative. 
It is easy to see that the definition of source terms provides the positiveness of the production of total entropy $S=S_{1}+S_{2}$, the equation for which can be obtained by a summation of the equations for partial entropies, namely

$$
\frac{\partial \rho S}{\partial t}+\frac{\partial\left(\rho S u+E_{j_{1}^{k}}+E_{j_{2}^{k}}\right)}{\partial x^{k}}=\Pi=\Pi_{1}+\Pi_{2}+\frac{1}{\tau^{(T)}} \frac{\left(E_{S_{1}}-E_{S_{2}}\right)^{2}}{E_{S_{1}} E_{S_{2}}} \geq 0
$$

Note that all coefficients in the source terms can be functions of the parameters of state.

As in the simplified model for isentropic two-phase flow (Sect. 2), an additional compatibility equation connected with the relative velocity vorticity (2.6) and (2.7) should be added to system (4.2).

The solution to system (4.2) satisfies an additional total energy conservation law, which can be derived by the summing up of all equations of this system multiplied by the corresponding factors: $E+\rho E_{\rho}-u_{l} u^{l} / 2-\alpha E_{\alpha}-c E_{c}-j_{1}^{k} E_{j_{1}^{k}}-j_{2}^{k} E_{j_{2}^{k}}-S_{1} E_{S_{1}}-S_{2} E_{S_{2}}, E_{\alpha}$, $E_{c}, u^{k}, \rho E_{w_{k}}, E_{j_{1}^{k}}, E_{j_{2}^{k}}, E_{S_{1}}, E_{S_{2}}$ (see Appendix). The equation itself has the following form:

$$
\frac{\partial \rho\left(E+\frac{u_{l} u^{l}}{2}\right)}{\partial t}+\frac{\partial\left(\rho u^{k}\left(E+\frac{u_{l} u^{l}}{2}+\frac{p}{\rho}+w^{l} E_{w^{l}}\right)+E_{c} E_{w_{k}}+E_{j_{i}^{k}} E_{S_{i}}\right)}{\partial x^{k}}=0 .
$$

Below we describe how to choose closing relationships, namely, equation of state and coefficients in the source terms.

4.2. Equation of state. Suppose that we know the internal energy

$$
e^{i}=e^{i}\left(\rho_{i}, s_{i}, j_{i}^{k}\right)
$$

for each phase as a function of its mass density $\rho_{i}$, specific entropy $s_{i}$ and specific thermal impulse $j_{i}^{k}$. The dependence on the thermal impulse $j_{i}^{k}$ can be taken as in Sect. 3.

$$
e^{i}=e_{0}^{i}\left(\rho_{i}, s_{i}\right)+A_{i} \frac{j_{j}^{k} j_{i}^{k}}{2}
$$

where $A_{i}$ is a constant.

As in Sect. 2 we take the equation of state for the mixture as a sum of specific internal energy and kinematic energy:

$$
E\left(\rho, \alpha, c, S_{1}, S_{2}, j_{1}^{k}, j_{2}^{k}, w^{k}\right)=e\left(\rho, \alpha, c, S_{1}, S_{2}, j_{1}^{k}, j_{2}^{k}\right)+c(1-c) \frac{w_{k} w^{k}}{2}
$$

The specific internal energy $e$ we define using known $e_{1}, e_{2}$ by the formula

$$
e\left(\rho, \alpha, c, S_{1}, S_{2}, j_{1}^{k}, j_{2}^{k}\right)=c_{1} e_{1}\left(\rho_{1}, s_{1}, j_{1}^{k}\right)+c_{2} e_{2}\left(\rho_{2}, s_{2}, j_{2}^{k}\right) .
$$


Furthermore, we will use a dependence of parameters of state for each individual phase on the parameters of state for the mixture. It can be derived from (4.1) and reads as follows:

$$
\begin{gathered}
u_{1}^{k}=u^{k}+(1-c) w^{k}, \quad u_{2}^{k}=u^{k}-c w^{k}, \quad \rho_{1}=\frac{c \rho}{\alpha}, \quad \rho_{2}=\frac{(1-c) \rho}{(1-\alpha)} \\
s_{1}=\frac{S_{1}}{c_{1}}, \quad s_{2}=\frac{S_{2}}{c_{2}}, \quad j_{1}^{k}=j_{1}^{k}, \quad j_{2}^{k}=j_{2}^{k} .
\end{gathered}
$$

Using these formulae we can derive the derivatives of the equation of state for the mixture $E_{\alpha}, E_{\rho}, E_{c}, E_{w^{k}}, E_{S_{1}}, E_{j_{1}^{k}}, E_{S_{2}}, E_{j_{2}^{k}}$ by the derivatives of equations of state for individual phases $e_{\rho_{1}}^{1}, e_{s_{1}}^{1}, e_{j_{1}^{k}}^{1}$ and $e_{\rho_{2}}^{2}, e_{s_{2}}^{2}, e_{j_{2}^{k}}^{2}$.

To do this we can use the following set of thermodynamic identities:

$$
\begin{gathered}
d e=d\left(c_{1} e^{1}+c_{2} e^{2}\right)=\left(e^{1}-e^{2}\right) d c+c_{1} d e^{1}+c_{2} d e^{2}, \\
d e^{1}=e_{\rho_{1}}^{1} d \rho_{1}+e_{s_{1}}^{1} d s_{1}+e_{j_{1}^{k}}^{1} d j_{1}^{k}, \quad d e^{2}=e_{\rho_{2}}^{2} d \rho_{2}+e_{s_{2}}^{2} d s_{2}+e_{j_{2}^{k}}^{2} d j_{2}^{k}, \\
d \rho_{1}=\frac{c_{1}}{\alpha_{1}} d \rho-\frac{\rho_{1}}{\alpha_{1}} d \alpha+\frac{\rho}{\alpha_{1}} d c, \quad d \rho_{2}=\frac{c_{2}}{\alpha_{2}} d \rho+\frac{\rho_{2}}{\alpha_{2}} d \alpha-\frac{\rho}{\alpha_{2}} d c \\
d s_{1}=\frac{1}{c_{1}} d S_{1}-\frac{s_{1}}{c_{1}} d c, \quad d s_{2}=\frac{1}{c_{2}} d S_{2}+\frac{s_{2}}{c_{2}} d c .
\end{gathered}
$$

These identities lead us to the final thermodynamic identity

$$
\begin{gathered}
d e=e_{\rho} d \rho+e_{\alpha} d \alpha+e_{c} d c+e_{S_{1}} d S_{1}+e_{S_{2}} d S_{2}+e_{j_{1}^{k}} d j_{1}^{k}+e_{j_{2}^{k}} d j_{2}^{k} \\
=\frac{1}{\rho^{2}}\left(\alpha_{1} \rho_{1}^{2} e_{\rho_{1}}^{1}+\alpha_{2} \rho_{2}^{2} e_{\rho_{2}}^{2}\right) d \rho+\frac{1}{\rho}\left(\rho_{1}^{2} e_{\rho_{1}}^{1}-\rho_{2}^{2} e_{\rho_{2}}^{2}\right) d \alpha \\
+\left(\left(e^{1}+\rho_{1} e_{\rho_{1}}^{1}-s_{1} e_{s_{1}}^{1}\right)-\left(e^{2}+\rho_{2} e_{\rho_{2}}^{2}-s_{2} e_{s_{2}}\right)\right) d c \\
+e_{s_{1}}^{1} d S_{1}+c_{1} e_{j_{1}^{k}}^{1} d j_{1}^{k}+e_{s_{2}}^{2} d S_{2}+c_{2} e_{j_{2}^{k}}^{2} d j_{2}^{k}
\end{gathered}
$$

from which we obtain

$$
\begin{aligned}
& E_{\rho}=\frac{1}{\rho^{2}}\left(\alpha_{1} \rho_{1}^{2} e_{\rho_{1}}^{1}+\alpha_{2} \rho_{2}^{2} e_{\rho_{2}}^{2}\right)=\frac{\alpha_{1} p^{1}+\alpha_{2} p^{2}}{\rho^{2}} \\
& E_{\alpha}=\frac{1}{\rho}\left(\rho_{1}^{2} e_{\rho_{1}}^{1}-\rho_{2}^{2} e_{\rho_{2}}^{2}\right)=\frac{p^{1}-p^{2}}{\rho}, \\
& E_{c}=\left(\left(e^{1}+p^{1} / \rho_{1}-s_{1} e_{s_{1}}^{1}\right)-\left(e^{2}+p^{2} / \rho_{2}-s_{2} e_{s_{2}}^{2}\right)\right)+(1-2 c) \frac{w_{k} w^{k}}{2} \\
& E_{S_{1}}=e_{s_{1}}^{1}=T^{1}, \quad E_{S_{2}}=e_{s_{2}}^{2}=T^{2}, \quad E_{j_{1}^{k}}=c_{1} e_{j_{1}^{k}}^{1}, \quad E_{j_{2}^{k}}=c_{2} e_{j_{2}^{k}}^{2} .
\end{aligned}
$$


Here $T^{1}, T^{2}$ are temperatures of phases. It is interesting to note that the derivative $e_{c}$ of the specific internal energy with respect to $c$ is simply the difference of phase chemical potentials $\mu^{i}$ :

$$
\begin{gathered}
e_{c}=\mu^{1}-\mu^{2}, \\
\mu^{i}=e^{i}+\frac{p^{i}}{\rho_{i}}-s_{i} T^{i} .
\end{gathered}
$$

Now we can write the system of governing equations in terms of individual parameters of state for each phase.

4.3. Governing equations in terms of individual parameters of state. The system of conservative balance equations (4.2) can be rewritten using individual parameters of each phase $\alpha_{1}, \alpha_{2}, \rho_{1}, \rho_{2}, u_{1}^{k}, u_{2}^{k}, s_{1}, s_{2}, j_{1}^{k}, j_{2}^{k}$ (recall that the volume fraction of the second phase can be determined via the volume fraction of the first phase: $\alpha_{2}=1-\alpha_{1}$ ). To do this it is necessary to use formulae (4.1), (4.3) and (4.6). The resulting system is as follows (note that we left the equation for $\alpha$ as in (4.2)):

$$
\begin{aligned}
& \frac{\partial\left(\alpha_{1} \rho_{1}+\alpha_{2} \rho_{2}\right)}{\partial t}+\frac{\partial\left(\alpha_{1} \rho_{1} u_{1}^{k}+\alpha_{2} \rho_{2} u_{2}^{k}\right)}{\partial x^{k}}=0, \\
& \frac{\partial \rho \alpha}{\partial t}+\frac{\partial \rho \alpha u^{k}}{\partial x^{k}}=-\phi, \\
& \frac{\partial \alpha_{1} \rho_{1}}{\partial t}+\frac{\partial \alpha_{1} \rho_{1} u_{1}^{k}}{\partial x^{k}}=-\psi, \\
& \frac{\partial\left(\alpha_{1} \rho_{1} u_{1}^{i}+\alpha_{2} \rho_{2} u_{2}^{i}\right)}{\partial t}+\frac{\partial\left(\alpha_{1} \rho_{1} u_{1}^{i} u_{1}^{k}+\alpha_{2} \rho_{2} u_{2}^{i} u_{2}^{k}+\alpha_{1} p^{1}+\alpha_{2} p^{2}\right)}{\partial x^{k}}=0, \\
& \frac{\partial\left(u_{1}^{k}-u_{2}^{k}\right)}{\partial t}+\frac{\partial\left(u_{1}^{i} u_{1}^{i} / 2-u_{2}^{i} u_{2}^{i} / 2+\mu^{1}-\mu^{2}\right)}{\partial x^{k}}=-e_{k l j} u^{l} \omega^{j}-\frac{1}{\rho} \lambda_{0}^{k}, \\
& \frac{\partial \rho j_{1}^{i}}{\partial t}+\frac{\partial\left(\rho j_{1}^{i} u^{k}+e_{s_{1}}^{1} \delta^{i k}\right)}{\partial x^{k}}=-\lambda_{1}^{i}, \\
& \frac{\partial \rho j_{2}^{i}}{\partial t}+\frac{\partial\left(\rho j_{2}^{i} u^{k}+e_{s_{2}}^{2} \delta^{i k}\right)}{\partial x^{k}}=-\lambda_{2}^{i}, \\
& \frac{\partial \alpha_{1} \rho_{1} s_{1}}{\partial t}+\frac{\partial\left(\alpha_{1} \rho_{1} s_{1} u^{k}+c_{1} e_{j_{1}^{k}}^{1}\right)}{\partial x^{k}}=\Pi_{1}-\pi_{1}, \\
& \frac{\partial \alpha_{2} \rho_{2} s_{2}}{\partial t}+\frac{\partial\left(\alpha_{2} \rho_{2} s_{2} u^{k}+c_{2} e_{j_{2}^{k}}^{2}\right)}{\partial x}=\Pi_{2}-\pi_{2} .
\end{aligned}
$$

As in Sect. 2, this system must be supplemented by the compatibility relations (2.6), (2.7) for the relative velocity vorticity $\omega_{j}$. 
The source terms in the above equations can also be written in terms of individual parameters of phases:

$$
\begin{gathered}
\phi=\frac{p_{1}-p_{2}}{\tau^{(p)}}, \quad \psi=\frac{\rho}{\tau^{(c)}}\left(\mu^{1}-\mu^{2}+\frac{1}{2}\left(c_{1}-c_{2}\right)\left(u_{1}^{i}-u_{2}^{i}\right)\left(u_{1}^{i}-u_{2}^{i}\right)\right), \\
\lambda_{0}^{k}=\chi_{00} c_{1} c_{2}\left(u_{1}^{k}-u_{2}^{k}\right)+\chi_{01} c_{1} e_{j_{1}^{k}}^{1}+\chi_{02} c_{2} e_{j_{2}^{k}}^{2}, \\
\lambda_{1}^{i}=\chi_{01} c_{1} c_{2}\left(u_{1}^{i}-u_{2}^{i}\right)+\chi_{11} c_{1} e_{j_{1}^{i}}^{1}, \quad \lambda_{2}^{i}=\chi_{02} c_{1} c_{2}\left(u_{1}^{i}-u_{2}^{i}\right)+\chi_{22} c_{2} e_{j_{2}^{i}}^{2} \\
\Pi_{1}=\frac{1}{T^{1}} \chi_{11} \sum_{k=1}^{3}\left(c_{1} e_{j_{1}^{k}}^{1}+\frac{\chi_{01}}{\chi_{11}} c_{1} c_{2}\left(u_{1}^{k}-u_{2}^{k}\right)\right)^{2} \\
+\frac{c_{1}}{T^{1}}\left(\chi_{00}-\frac{\chi_{01}^{2}}{\chi_{11}}-\frac{\chi_{02}^{2}}{\chi_{22}}\right) \sum_{k=1}^{3}\left(c_{1} c_{2}\left(u_{1}^{k}-u_{2}^{k}\right)\right)^{2}+\frac{c_{1}}{T^{1}} \frac{\left(p^{1}-p^{2}\right)^{2}}{\rho \tau^{(p)}} \\
+\frac{\rho c_{1}}{T^{1}} \frac{\left(\mu^{1}-\mu^{2}+\left(c_{1}-c_{2}\right)\left(u_{1}^{i}-u_{2}^{i}\right)\left(u_{1}^{i}-u_{2}^{i}\right) / 2\right)^{2}}{\tau^{(c)}} \\
\Pi_{2}=\frac{1}{T^{2}} \chi_{22} \sum_{k=1}^{3}\left(c_{2} e_{j_{2}^{k}}^{2}+\frac{\chi_{02}}{\chi_{22}} c_{1} c_{2}\left(u_{1}^{k}-u_{2}^{k}\right)\right)^{2} \\
+\frac{c_{2}}{T^{2}}\left(\chi_{00}-\frac{\chi_{01}^{2}}{\chi_{11}}-\frac{\chi_{02}^{2}}{\chi_{22}}\right) \sum_{k=1}^{3}\left(c_{1} c_{2}\left(u_{1}^{k}-u_{2}^{k}\right)\right)^{2}+\frac{c_{2}}{T^{2}} \frac{\left(p^{1}-p^{2}\right)^{2}}{\rho \tau^{(p)}} \\
+\frac{\rho c_{2}}{T^{2}} \frac{\left(\mu^{1}-\mu^{2}+\left(c_{1}-c_{2}\right)\left(u_{1}^{i}-u_{2}^{i}\right)\left(u_{1}^{i}-u_{2}^{i}\right) / 2\right)^{2}}{\tau^{(c)}} \\
\pi_{1}=\frac{1}{\tau^{(T)}} \frac{T^{1}-T^{2}}{T^{1}}, \quad \pi_{2}=\frac{1}{\tau^{(T)}} \frac{T^{2}-T^{1}}{T^{2}}
\end{gathered}
$$

Finally we can rewrite the total mixture entropy balance Eq. (4.4) and energy conservation law (4.5) in terms of individual parameters:

$$
\begin{gathered}
\frac{\partial\left(\alpha_{1} \rho_{1} s_{1}+\alpha_{2} \rho_{2} s_{2}\right)}{\partial t}+\frac{\partial\left(\left(\alpha_{1} \rho_{1} s_{1}+\alpha_{2} \rho_{2} s_{2}\right) u^{k}+c_{1} e_{j_{1}^{k}}^{1}+c_{2} e_{j_{2}^{k}}^{2}\right)}{\partial x^{k}} \\
=\Pi=\Pi_{1}+\Pi_{2}+\frac{1}{\tau^{(T)}} \frac{\left(T^{1}-T^{2}\right)^{2}}{T^{1} T^{2}} \geq 0 \\
\frac{\partial}{\partial t}\left(\alpha_{1} \rho_{1}\left(e^{1}+\frac{u_{1}^{i} u_{1}^{i}}{2}\right)+\alpha_{2} \rho_{2}\left(e^{2}+\frac{u_{2}^{i} u_{2}^{i}}{2}\right)\right)+\frac{\partial}{\partial x^{k}}\left(\alpha_{1} \rho_{1} u_{1}^{k}\left(e^{1}+\frac{p^{1}}{\rho_{1}}+\frac{u_{1}^{i} u_{1}^{i}}{2}\right)\right. \\
\left.+\alpha_{2} \rho_{2} u_{2}^{k}\left(e^{2}+\frac{p^{2}}{\rho_{2}}+\frac{u_{2}^{i} u_{2}^{i}}{2}\right)+c_{1} T^{1} e_{j_{1}^{k}}^{1}+c_{2} T^{2} e_{j_{2}^{k}}^{2}\right)=0 .
\end{gathered}
$$

It is interesting to note that the difference of chemical potentials of phases is presented in the system (4.7) twice, namely in the relative velocity flux and in the source term that is responsible for the phase transition. The thermodynamic theory uses equality of phase 
chemical potentials as phase equilibrium condition [14. In our model such an equality is also responsible for the kinematic equilibrium of phases. In other words, the chemical potentials difference causes the relative motion of the phases.

There is a set of coefficients $\chi_{00}, \chi_{01}, \chi_{02}, \chi_{11}, \chi_{22}$ in the source terms $\lambda_{0}^{i}, \lambda_{1}^{i}, \lambda_{2}^{i}$ that should be defined. We take

$$
\begin{gathered}
\chi_{00}=\zeta+\frac{\chi_{01}^{2}}{\chi_{11}}+\frac{\chi_{02}^{2}}{\chi_{22}}, \quad \chi_{01}=-\frac{\rho s_{1}}{c_{1} \kappa_{1}}, \quad \chi_{02}=\frac{\rho s_{2}}{c_{2} \kappa_{2}}, \\
\chi_{11}=\frac{1}{c_{1} \kappa_{1}}, \quad \chi_{22}=\frac{1}{c_{2} \kappa_{2}},
\end{gathered}
$$

where $\zeta$ is an interfacial friction coefficient, and $\kappa_{1}, \kappa_{2}$ are the coefficients of thermal conductivity for phases.

The motivation for this choice is caused by the requirement related to phase entropy fluxes in the case of negligible thermal conductivity. This requirement can be formulated in the following way: if the phase heat transfer characteristic times are large in comparison to the characteristic time of the process under consideration (in other words, if $\kappa_{1}, \kappa_{2} \rightarrow 0$ ), then the partial phase entropy fluxes are

$$
\alpha_{1} \rho_{1} s_{1} u_{1}^{k}, \quad \alpha_{2} \rho_{2} s_{2} u_{2}^{k} .
$$

Note that only interfacial friction and thermal conductivity coefficients are required to define $\chi_{00}, \chi_{01}, \chi_{02}, \chi_{11}, \chi_{22}$ for each concrete two-phase mixture. The consequence of the above choice of coefficients $\chi_{i j}$ is provided in the next section, in which the diffusive limit of the heat flux is discussed. It turns out that the equations, which are derived assuming that $\kappa_{i}$ are sufficiently small, look similar to the Baer-Nunziato equations in which the phase parabolic thermal conductivities are taken into account.

4.4. Diffusive heat transfer limit. In Sect. 3 it was observed that the hyperbolic heat transfer equations give a parabolic approximation of heat transfer processes if the heat flux relaxation time is small. In case of two-phase flow we have two interacting heat transfer processes for each phase with two different heat flux relaxation times. Here we study the relaxation limit in both phases assuming that two heat flux relaxation times are sufficiently small. We also suppose that the characteristic scale of the process is much larger than the relaxation times.

Let us consider the equations from the system (4.7) for phase thermal impulses:

$$
\frac{\partial \rho j_{m}^{i}}{\partial t}+\frac{\partial\left(\rho j_{m}^{i} u^{k}+e_{s_{m}}^{m} \delta_{i k}\right)}{\partial x^{k}}=-\lambda_{m}^{i}, \quad m=1,2,
$$

where $\lambda_{m}^{i}$ is defined by (4.8) with coefficients (4.11).

Following the assumption concerning the smallness of $\kappa_{1}, \kappa_{2}$ and the characteristic time scale of processes, we can neglect the time derivative and convective term in the equation for $q_{m}^{i}$. The resulting equation is

$$
\lambda_{m}^{k}=-\frac{\partial e_{s_{m}}^{m}}{\partial x^{k}}=-\frac{\partial T^{m}}{\partial x^{k}}, \quad m=1,2
$$


Now with use of the formulae for coefficients (4.8), (4.11), we obtain

$$
\begin{gathered}
c_{1} e_{j_{1}^{k}}^{1}=\rho s_{1} c_{1} c_{2}\left(u_{1}^{k}-u_{2}^{k}\right)-c_{1} \kappa_{1} \frac{\partial T^{1}}{\partial x^{k}}, \\
c_{2} e_{j_{2}^{k}}^{2}=-\rho s_{2} c_{1} c_{2}\left(u_{1}^{k}-u_{2}^{k}\right)-c_{2} \kappa_{2} \frac{\partial T^{1}}{\partial x^{k}} .
\end{gathered}
$$

From the above formulae we see that in the case of considerable relative motion of phases, the leading terms in dissipative entropy fluxes are connected to the relative velocity whereas the parabolic terms can be negligible due to the smallness of $\kappa_{1}, \kappa_{2}$.

Finally, the phases entropies balance equations are transformed to

$$
\begin{aligned}
& \frac{\partial \alpha_{1} \rho_{1} s_{1}}{\partial t}+\frac{\partial \alpha_{1} \rho_{1} s_{1} u_{1}^{k}}{\partial x^{k}}-\frac{\partial}{\partial x^{k}} c_{1} \kappa_{1} \frac{\partial T^{1}}{\partial x^{k}}=\Pi_{1}-\pi_{1}, \\
& \frac{\partial \alpha_{2} \rho_{2} s_{2}}{\partial t}+\frac{\partial \alpha_{2} \rho_{2} s_{2} u_{2}^{k}}{\partial x^{k}}-\frac{\partial}{\partial x^{k}} c_{2} \kappa_{2} \frac{\partial T^{2}}{\partial x}=\Pi_{2}-\pi_{2} .
\end{aligned}
$$

Expressions (4.12) should be also substituted into entropy production terms $\Pi_{k}, \pi_{k}$, which are defined by (4.8) and become ( $\pi_{k}$ does not change)

$$
\begin{gathered}
\Pi_{1}=\frac{c_{1} \kappa_{1}}{T^{1}} \sum_{k=1}^{3}\left(\frac{\partial T^{1}}{\partial x^{k}}\right)^{2}+\frac{c_{1} \zeta}{T^{1}} \sum_{k=1}^{3}\left(c_{1} c_{2}\left(u_{1}^{k}-u_{2}^{k}\right)\right)^{2}+\frac{c_{1}}{T^{1}} \frac{\left(p^{1}-p^{2}\right)^{2}}{\rho \tau^{(p)}} \\
+\frac{\rho c_{1}}{T^{1}} \frac{\left(\mu^{1}-\mu^{2}+\left(c_{1}-c_{2}\right)\left(u_{1}^{i}-u_{2}^{i}\right)\left(u_{1}^{i}-u_{2}^{i}\right) / 2\right)^{2}}{\tau^{(c)}}, \\
\Pi_{2}=\frac{c_{2} \kappa_{2}}{T^{2}} \sum_{k=1}^{3}\left(\frac{\partial T^{2}}{\partial x^{k}}\right)^{2}+\frac{c_{2} \zeta}{T^{2}} \sum_{k=1}^{3}\left(c_{1} c_{2}\left(u_{1}^{k}-u_{2}^{k}\right)\right)^{2}+\frac{c_{2}}{T^{2}} \frac{\left(p^{1}-p^{2}\right)^{2}}{\rho \tau^{(p)}} \\
+\frac{\rho c_{2}}{T^{2}} \frac{\left(\mu^{1}-\mu^{2}+\left(c_{1}-c_{2}\right)\left(u_{1}^{i}-u_{2}^{i}\right)\left(u_{1}^{i}-u_{2}^{i}\right) / 2\right)^{2}}{\tau^{(c)}}, \\
\pi_{1}=\frac{1}{\tau^{(T)}} \frac{T^{1}-T^{2}}{T^{1}}, \quad \pi_{2}=\frac{1}{\tau^{(T)}} \frac{T^{2}-T^{1}}{T^{2}} .
\end{gathered}
$$

So the diffusive limit leads to the traditional parabolic equations for phase heat transfer.

It is interesting to look at the transformed relative velocity equation, because the original balance law contains a source term in which thermal impulses are included. Substituting (4.12) into the source term in the equation for the relative velocity, we obtain

$$
\begin{aligned}
& \frac{\partial\left(u_{1}^{k}-u_{2}^{k}\right)}{\partial t}+\frac{\partial\left(u_{1}^{i} u_{1}^{i} / 2-u_{2}^{i} u_{2}^{i} / 2+\mu^{1}-\mu^{2}\right)}{\partial x^{k}} \\
= & -s_{1} \frac{\partial T^{1}}{\partial x^{k}}+s_{2} \frac{\partial T^{2}}{\partial x^{k}}-\zeta c_{1} c_{2}\left(u_{1}^{k}-u_{2}^{k}\right)-e_{k l j} u_{k} \omega_{j} .
\end{aligned}
$$

We see that, although the heat flux relaxation times are small, the influence of heat transfer on the relative motion is finite.

The latter equation can be transformed to

$$
\left(\frac{\partial u_{1}^{k}}{\partial t}+u_{1}^{i} \frac{\partial u_{1}^{k}}{\partial x^{i}}+\frac{1}{\rho_{1}} \frac{\partial p_{1}}{\partial x^{k}}\right)-\left(\frac{\partial u_{2}^{k}}{\partial t}+u_{2}^{i} \frac{\partial u_{2}^{k}}{\partial x^{i}}+\frac{1}{\rho_{2}} \frac{\partial p_{2}}{\partial x^{k}}\right)=-\zeta c_{1} c_{2}\left(u_{1}^{k}-u_{2}^{k}\right),
$$


which can be used to derive individual phase momentum equations. It turns out that they are identical to the phase momentum equations derived in the Sect. 2.

So we conclude that the diffusive limit of the system for two-phase flow (4.7) consists of the balance laws for phase volume fraction $\alpha$, mass and momentum balance equations for two phases, and balance equations for partial entropies (4.13). The full system is similar to the equations of the Baer-Nunziato model, in which the phase thermal conductivity is taken into account:

$$
\begin{aligned}
& \frac{\partial \rho \alpha_{1}}{\partial t}+\frac{\partial \rho \alpha_{1} u^{k}}{\partial x^{k}}=-\phi, \\
& \frac{\partial \alpha_{1} \rho_{1}}{\partial t}+\frac{\partial \alpha_{1} \rho_{1} u_{1}^{k}}{\partial x^{k}}=-\psi, \\
& \frac{\partial \alpha_{2} \rho_{2}}{\partial t}+\frac{\partial \alpha_{2} \rho_{2} u_{2}^{k}}{\partial x^{k}}=\psi, \\
& \frac{\partial \alpha_{1} \rho_{1} u_{1}^{i}}{\partial t}+\frac{\partial \alpha_{1} \rho_{1} u_{1}^{i} u_{1}^{k}}{\partial x^{k}}+\frac{\partial \alpha_{1} p^{1}}{\partial x^{i}}=\hat{p}_{I} \frac{\partial \alpha_{1}}{\partial x^{i}}+f_{i}+\hat{\zeta}\left(u_{2}^{i}-u_{1}^{i}\right)-\left(c_{2} u_{1}^{i}+c_{1} u_{2}^{i}\right) \psi, \\
& \frac{\partial \alpha_{2} \rho_{2} u_{2}^{i}}{\partial t}+\frac{\partial \alpha_{2} \rho_{2} u_{2}^{i} u_{2}^{k}}{\partial x^{k}}+\frac{\partial \alpha_{2} p^{2}}{\partial x^{i}}=\hat{p}_{I} \frac{\partial \alpha_{2}}{\partial x^{i}}-f_{i}-\hat{\zeta}\left(u_{2}^{i}-u_{1}^{i}\right)+\left(c_{2} u_{1}^{i}+c_{1} u_{2}^{i}\right) \psi, \\
& \frac{\partial \alpha_{1} \rho_{1} s_{1}}{\partial t}+\frac{\partial \alpha_{1} \rho_{1} s_{1} u_{1}^{k}}{\partial x^{k}}-\frac{\partial}{\partial x^{k}} c_{1} \kappa_{1} \frac{\partial T^{1}}{\partial x^{k}}=\Pi_{1}-\pi_{1}, \\
& \frac{\partial \alpha_{2} \rho_{2} s_{2}}{\partial t}+\frac{\partial \alpha_{2} \rho_{2} s_{2} u_{2}^{k}}{\partial x^{k}}-\frac{\partial}{\partial x^{k}} c_{2} \kappa_{2} \frac{\partial T^{2}}{\partial x^{k}}=\Pi_{2}-\pi_{2},
\end{aligned}
$$

where $\phi, \psi$ are defined by (4.8), $\Pi_{i}, \pi_{i}$ are defined by (4.14), $\hat{p}_{I}$ is defined by (2.15), $f_{i}$ is defined by (2.16), and $\hat{\zeta}$ is defined by (2.14).

Note that the phase entropy balance equations are presented in the latter system, but we can also derive phase energy balance equations, which are used in the Baer-Nunziato type model and look as follows:

$$
\begin{gathered}
\frac{\partial}{\partial t}\left(\alpha_{1} \rho_{1}\left(e^{1}+\frac{u_{1}^{i} u_{1}^{i}}{2}\right)\right)+\frac{\partial}{\partial x^{k}}\left(\alpha_{1} \rho_{1} u_{1}^{k}\left(e^{1}+\frac{u_{1}^{i} u_{1}^{i}}{2}\right)+\alpha_{1} p^{1} u_{1}^{k}-c_{1} \kappa_{1} T^{1} \frac{\partial T^{1}}{\partial x^{k}}\right) \\
=-p^{1} \frac{\partial \alpha_{1}}{\partial t}+\left(\hat{p}_{I}-p^{1}\right) u_{1}^{k} \frac{\partial \alpha_{1}}{\partial x^{k}}+u_{1}^{k} f_{k}+\hat{\zeta}\left(u_{2}^{i}-u_{1}^{i}\right) u_{1}^{i} \\
\quad-\left(\mu_{1}+\left(c_{2}-c_{1}\right) \frac{u_{1}^{2}}{2}+c_{1} u_{1} u_{2}\right) \psi+T^{1} \Pi_{1}-\frac{T^{1}-T^{2}}{\tau^{(T)}}, \\
\frac{\partial}{\partial t}\left(\alpha_{2} \rho_{2}\left(e^{2}+\frac{u_{2}^{i} u_{2}^{i}}{2}\right)\right)+\frac{\partial}{\partial x^{k}}\left(\alpha_{2} \rho_{2} u_{2}^{k}\left(e^{2}+\frac{u_{2}^{i} u_{2}^{i}}{2}\right)+\alpha_{2} p^{2} u_{2}^{k}-c_{2} \kappa_{2} T^{2} \frac{\partial T^{2}}{\partial x^{k}}\right) \\
=-p^{2} \frac{\partial \alpha_{2}}{\partial t}+\left(\hat{p}_{I}-p^{2}\right) u_{2}^{k} \frac{\partial \alpha_{2}}{\partial x^{k}}-u_{2}^{k} f_{k}-\hat{\zeta}\left(u_{2}^{i}-u_{1}^{i}\right) u_{2}^{i} \\
+\left(\mu_{2}-\left(c_{2}-c_{1}\right) \frac{u_{2}^{2}}{2}+c_{2} u_{1} u_{2}\right) \psi+T^{2} \Pi_{2}-\frac{T^{2}-T^{1}}{\tau^{(T)}} .
\end{gathered}
$$


So the diffusive limit of the proposed conservative equations for two-phase flow can be written in the form of the Baer-Nunziato type equations, which are not in conservative form.

5. Conclusions. A system of governing equations for two-phase compressible flow with two different pressures and temperatures of phases has been proposed. The derivation of the system is based on the formalism of thermodynamically compatible systems of conservation laws and on extended irreversible thermodynamics principles. The system is hyperbolic and is written in conservation-law form.

The phase interaction is modelled by the source terms added to the conservation equations and includes the phase pressure relaxation, phase transition, interfacial friction and phase temperatures equalizing.

The diffusive limit in the heat transfer equations in the proposed model can be transformed to the well-known Baer-Nunziato type of governing equations for two-phase flow in which traditional Fourier thermal conductivity is taken into account.

Because of its conservation form the presented model seems to be promising in applying known mathematical means and the development and application of modern numerical methods for studying different problems.

Appendix A. Thermodynamically compatible system of balance laws generating two-phase flow models. A Thermodynamically Compatible Systems theory has been applied to the formulation of general classes of conservation equations for the processes in complicated media in the presence of electromagnetic fields and external forces; see, for example, 11, 12, 18. This theory gives an elegant way to write the system of conservation laws in terms of generating potentials and variables. Such a formulation is based on the requirement for the system to be thermodynamically compatible; in other words, the complete system admits an additional conservation law correponding to the first law of thermodynamics. Each system written in terms of canonical variables and generating potentials can be reduced to a symmetric hyperbolic system, and together with its conservative form, it allows us to apply known mathematical means to study solutions of various initial-boundary problems. In 11, 21 the class of thermodynamically compatible systems generated by only one potential has been formulated. This class includes many systems of governing equations of continuum mechanics. If dissipation is not included into the governing equations, then the equations can be derived by variational principles in Lagrangean coordinates 10. After passing to Eulerean coordinates, dissipative processes can be included in the governing equations. A concrete system of continuum mechanics can be derived from the thermodynamically compatible system by assigning a physical meaning to the canonical variables and special choice of generating potential.

In this paper the governing equations of two-phase flow are designed on the basis of representatives of the class of thermodynamically compatible systems presented below. Note that we do not consider here possible dissipative processes. We give this description only to give an explanation of how the equations of the paper have been derived. Details 
concerning the inclusion of dissipation can be found in [22].

$$
\begin{aligned}
& \frac{\partial L_{q_{i}}}{\partial t}+\frac{\partial\left(u_{k} L\right)_{q_{i}}}{\partial x_{k}}=0, \quad i=1,2, \\
& \frac{\partial L_{u_{i}}}{\partial t}+\frac{\partial\left[\left(u_{k} L\right)_{u_{i}}+z_{k} L_{z_{i}}-\delta_{i k} z_{\alpha} L_{z_{\alpha}}\right]}{\partial x_{k}}=0, \\
& \frac{\partial L_{z_{k}}}{\partial t}+\frac{\partial\left(u_{\alpha} L_{z_{\alpha}}+n\right)}{\partial x_{k}}=0, \\
& \frac{\partial L_{n}}{\partial t}+\frac{\partial\left(u_{k} L_{n}+z_{k}\right)}{\partial x_{k}}=0, \\
& \frac{\partial L_{\theta^{n}}}{\partial t}+\frac{\partial\left(\left(u_{k} L\right)_{\theta^{n}}+v_{k}^{n}\right)}{\partial x_{k}}=0, \quad n=1,2, \\
& \frac{\partial L_{v_{i}^{n}}}{\partial t}+\frac{\partial\left(\left(u_{k} L\right)_{v_{i}^{n}}+\theta \delta_{i k}\right)}{\partial x_{k}}=0, \quad n=1,2 \\
& \frac{\partial L_{z_{k}}}{\partial x_{\alpha}}-\frac{\partial L_{z_{\alpha}}}{\partial x_{k}}=0 .
\end{aligned}
$$

Here $L\left(q_{i}, u_{i}, z_{k}, n, \theta^{n}, v_{i}^{n}\right)$ is the generating potential assumed to be a convex function. The last steady equation must be added to the system in order to provide the compatibility of the system (A.1). Actually this steady equation is a consequence of the equation for $L_{z_{k}}$ and can be obtained by differentiating the equation for $L_{z_{k}}$ with respect to $x_{\alpha}$ and subtracting the equation for $L_{z_{\alpha}}$ differentiated with respect to $x_{k}$. After that we obtain

$$
\frac{\partial}{\partial t}\left(\frac{\partial L_{z_{k}}}{\partial x_{\alpha}}-\frac{\partial L_{z_{\alpha}}}{\partial x_{k}}\right)=0
$$

and if in the initial data $\partial L_{z_{k}} / \partial x_{\alpha}-\partial L_{z_{\alpha}} / \partial x_{k}=0$, then this equality holds for $t>0$ every time.

As was noted for the thermodynamically compatible system, an additional (energy) conservation law exists. In our case it looks as follows:

$$
\begin{gathered}
\frac{\partial}{\partial t}\left(q_{i} L_{q_{i}}+u_{i} L_{u_{i}}+z_{k} L_{z_{k}}+n L_{n}+\theta^{n} L_{\theta^{n}}+v_{i}^{n} L_{v_{i}^{n}}-L\right) \\
+\frac{\partial}{\partial x_{k}}\left(u_{k}\left(q_{i} L_{q_{i}}+u_{i} L_{u_{i}}+n L_{n}+\theta^{n} L_{\theta^{n}}+v_{i}^{n} L_{v_{i}^{n}}\right)+u_{l} z_{k} L_{z_{l}}+z_{k} n+\theta^{n} v_{k}^{n}\right)=0 .
\end{gathered}
$$

To derive this conservation law it is necessary to sum all equations of the system (A.1) multiplied respectively by

$$
q_{i}, u_{i}, z_{k}, n, \theta^{n}, v_{i}^{n}, 2 u_{i} z_{k} .
$$

Finally, the symmetric hyperbolic system can be derived from (A.1). To perform this we rewrite the evolution equations in the equivalent form, which is simply obtained by adding $z_{k}\left(\frac{\partial L_{z_{k}}}{\partial x_{i}}-\frac{\partial L_{z_{i}}}{\partial x_{k}}\right)=0$ to the equation for $u_{i}$ and $u_{i}\left(\frac{\partial L_{z_{k}}}{\partial x_{i}}-\frac{\partial L_{z_{i}}}{\partial x_{k}}\right)=0$ to the 
equation for $z_{k}$. After that we obtain the following system:

$$
\begin{aligned}
& \frac{\partial L_{q_{i}}}{\partial t}+\frac{\partial\left(u_{k} L\right)_{q_{i}}}{\partial x_{k}}=0, \\
& \frac{\partial L_{u_{i}}}{\partial t}+\frac{\partial\left(u_{k} L\right)_{u_{i}}}{\partial x_{k}}+L_{z_{i}} \frac{\partial z_{k}}{\partial x_{k}}-L_{z_{\alpha}} \frac{\partial z_{\alpha}}{\partial x_{i}}=0, \\
& \frac{\partial L_{n}}{\partial t}+\frac{\partial\left(u_{k} L\right)_{n}}{\partial x_{k}}+\frac{\partial z_{k}}{\partial x_{k}}=0, \\
& \frac{\partial L_{z_{l}}}{\partial t}+\frac{\partial\left(u_{k} L\right)_{z_{l}}}{\partial x_{k}}+L_{z_{\alpha}} \frac{\partial u_{\alpha}}{\partial x_{l}}-L_{z_{l}} \frac{\partial u_{k}}{\partial x_{k}}+\frac{\partial n}{\partial x_{l}}=0 . \\
& \frac{\partial L_{\theta^{n}}}{\partial t}+\frac{\partial\left(u_{k} L\right)_{\theta}}{\partial x_{k}}+\frac{\partial v_{k}^{n}}{\partial x_{k}}=0, \\
& \frac{\partial L_{v_{k}^{n}}}{\partial t}+\frac{\partial\left(u_{k} L\right)_{v_{k}^{n}}}{\partial x_{k}}+\delta_{i k} \frac{\partial \theta^{n}}{\partial x_{k}}=0 .
\end{aligned}
$$

It is clear that the quasilinear form of the latter system is symmetric, and if the generating potential $L$ is a convex function, then the system is symmetric hyperbolic.

This system is a prototype of the generating system (4.2) of Subsect. 4.1. Actually, in order to give a physical meaning to the above formal thermodynamically compatible system, we have to identify all variables as physical variables and define the generating potential $L$. One can prove that the system (4.2) can be obtained taking the derivatives of generating potential as

$$
L_{q_{1}}=\rho, \quad L_{q_{2}}=\rho \alpha, \quad L_{u_{i}}=\rho u_{i}, L_{n}=\rho c, \quad L_{z_{k}}=w^{k}, \quad L_{\theta^{n}}=\rho S_{n}, \quad L_{v_{k}^{n}}=\rho j_{k}^{n},
$$

the variables as

$$
\begin{gathered}
q_{1}=E+\rho E_{\rho}-\frac{u_{i} u_{i}}{2}-\alpha E_{\alpha}-c E_{c}-j_{k}^{n} E_{j_{k}^{n}}-\theta^{n} E_{\theta^{n}}, \quad q_{2}=E_{\alpha}, \quad u^{i}, \\
n=E_{c}, \quad z_{l}=\rho E_{w^{l}}, \quad \theta^{n}=E_{S_{n}}, \quad v_{k}^{n}=E_{j_{k}^{n}},
\end{gathered}
$$

and finally the generating potential as

$$
L=\rho^{2} E_{\rho}-\rho w^{k} E_{w^{k}} .
$$

An explanation regarding how to incorporate some kinds of dissipative processes into the system (A.1) can be found in [22].

Acknowledgement. The paper is based on research partially performed during the participation of the first and third authors in the program Nonlinear Hyperbolic Waves in Phase Dynamics and Astrophysics at the Isaac Newton Institute for Mathematical Sciences, University of Cambridge. The third author acknowledges the support provided by the Isaac Newton Institute for Mathematical Sciences, University of Cambridge, UK, as co-organizer of the six-month program on Nonlinear Hyperbolic Waves in Phase Dynamics and Astrophysics, January to July 2003, and the associated EPSRC senior visiting fellowship, grant GR N09276. The first author acknowledges the Italian Ministry of Education and Research (MIUR) for the financial support provided during his stay as a visiting professor at the Department of Civil and Environmental Engineering, University of Trento. 


\section{REFERENCES}

[1] N. Andrianov, G. Warnecke, The Riemann problem for the Baer-Nunziato two-phase flow model. J. Comput. Physics 195 (2004), 434-464. MR2046106 (2004m:76165)

[2] M. Baer, J. Nunziato, A two-phase mixture theory for the deflagration-to-detonation transition $(D D T)$ in reactive granular materials. Int. J. Multiphase Flow 12 (1986), 861-889.

[3] J. Bdzil, R. Menikoff, S. Son S, et al., Two-phase modeling of deflagration-to-detonation transition in granular materials: a critical examination of modeling issues. Phys. Fluids 11 (1999), 378-402.

[4] G.Q. Chen, C.D. Levermore, T.-P. Liu, Hyperbolic conservation laws with stiff relaxation terms and entropy. Comm. Pure Appl. Math. 47 (1994), 787-830. MR.1280989 (95h:35133)

[5] S.R. de Groot, P. Mazur, Non-equilibrium Thermodynamics. Dover (1984).

[6] D.A. Drew, R.T. Lahey, Jr., The virtual mass and lift force on a sphere in rotating and straining inviscid flow. Int. J. Multiphase Flow 13 (1987), 113-121.

[7] K.O. Friedrichs, P.D. Lax, Systems of conservation equations with a convex extension. Proc. Nat. Acad. Sci. USA 68 (1971), 1686-1688. MR0285799 (44:3016)

[8] S. Gavrilyuk, R. Saurel, Mathematical and numerical modeling of two-phase compressible flows with micro-inertia. J. Comput. Phys. 175 (2002), 326-360. MR1877822 (2002i:76110)

[9] S.K. Godunov, An interesting class of quasilinear systems. Soviet Math. Dokl. 2 (1961), 947-949.

[10] S.K. Godunov, T.Yu. Mikhailova, E.I. Romenski, Systems of thermodynamically consistent conservation laws that are invariant under rotations. Siberian Math. J. 37 (1996), 690-705. MR1643366 (99g:35078)

[11] S.K. Godunov, E. Romensky, Thermodynamics, conservation laws and symmetric forms of differential equations in mechanics of continuous media, in Computational Fluid Dynamics Review 1995. John Wiley \& Sons, New York, (1995), 19-31.

[12] S.K. Godunov, E. Romenski, Elements of continuum mechanics and conservation laws. Kluwer Academic/Plenum Publishers, New York (2003). MR 1999156 (2005e:74004)

[13] H. Gouin, S. Gavrilyuk, Hamilton's principle and Rankine-Hugoniot conditions for general motions of mixtures. Meccanica 34 (1999), 39-47. MR1683706 (2000a:76008)

[14] L.D. Landau, E.M. Lifshitz, Statistical Physics, Part 1. Pergamon, 3rd Ed. (1994).

[15] T.P. Liu, Hyperbolic conservation laws with relaxation. Comm. Math. Phys. 108 (1987), 153-175. MR0872145(88f:35092)

[16] C.A. Lowe, Two-phase shock-tube problems and numerical methods of solution. J. Comput. Phys. 204 (2005), 598-632. MR.2131855 (2006a:76067)

[17] A.N. Malyshev, E.I. Romenski, Hyperbolic equations for heat conduction. Global solvability of the Cauchy problem. Siberian Math. J. 27 (1986), 128-134. MR0873716 (88b:35128)

[18] I. Müller, T. Ruggeri, Rational extended thermodynamics. Springer-Verlag, New York, (1998). MR.1632151(99h:80001)

[19] A.D. Resnyansky, A thermodynamically complete model for simulation of one-dimensional multiphase flows. DSTO System Sciences Laboratory, Edinburgh South Australia, Preprint DSTO-TR1510 (2003).

[20] A.D. Resnyansky, G. Katselis, E.I. Romensky, A.E. Wildegger-Gaissmaier, Laboratory study of the blast and fragmentation warheads. 21st Int. Symposium on Balistics, Adelaide, South Australia, 19-24 April, 2004, CD-ROM Proceedings.

[21] E. Romensky, Hyperbolic systems of thermodynamically compatible conservation laws in continuum mechanics. Math. Comput. Modelling 28 (1998), 115-130. MR1662561 (99k:73027)

[22] E. Romensky, Thermodynamics and hyperbolic systems of balance laws in continuum mechanics, in Godunov Methods: theory and applications, Kluwer Academic/Plenum publishers, (2001), 745-761. MR 1963637 (2004a:74001)

[23] E. Romenski, E.F. Toro, Compressible two-phase flow models: two-pressure models and numerical methods. Computational Fluid Dynamics Journal 13 (2004), 403-416.

[24] R. Saurel, R. Abgrall, A multiphase Godunov method for compressible multifluid and multiphase flows. J. Comput. Phys. 150 (1999), 425-467. MR.1684902 (99m:76097)

[25] D. Serre, Sur le principe variationnel des équations de la mécanique des fluides parfaits. Modél. Math. Anal. Numér. 27 (1993), 739-758. MR1246997 (94j:76056)

[26] C. Truesdell, Rational Thermodynamics. McGraw-Hill, New York, (1969). MR0366236 (51:2484) 